\title{
Efeitos de planos nutricionais e de fontes de metionina sobre o desempenho, rendimento e composição de carcaças de frangos de corte
}

[Effects of nutritional plans and methionine sources on broilers performance, carcass yield, and carcass composition]

\author{
R.S. Leite $^{1}$, J.S.R. Rocha ${ }^{1}$, B.C. Michel1 ${ }^{1}$, L.J.C. Lara $^{1}$, E.A. Ornelas $^{2}$, S.V. Cançado ${ }^{1 *}$, N.C. Baião ${ }^{1}$ \\ ${ }^{1}$ Escola de Veterinária - UFMG \\ Caixa Postal 567 \\ 30123-970 - Belo Horizonte, MG \\ ${ }^{2}$ LANAGRO-MG/MAPA - Pedro Leopoldo, MG
}

\begin{abstract}
RESUMO
Para avaliar os efeitos de dois diferentes planos nutricionais de aminoácidos sulfurados totais (AAST) e de duas fontes de metionina nas rações de frangos de corte, foi realizado um experimento com 840 frangos machos, da linhagem agRoss 308. Os tratamentos foram definidos pelos planos nutricionais de AAST (normal e reduzido) e pelas fontes de metionina (DL-metionina e metionina hidroxianáloga MHA) usadas nas rações. O delineamento experimental foi o inteiramente ao acaso, em arranjo fatorial $2 \times 2$, (duas fontes de metionina $\mathrm{x}$ dois planos nutricionais de AAST). Não foram encontradas diferenças entre os tratamentos para o consumo de ração, ganho de peso, viabilidade, rendimento de carcaça, porcentagem de peito e composição das carcaças e vísceras. As aves que receberam as rações com o plano normal de AAST apresentaram melhor conversão alimentar, e as que receberam ração contendo DL-metionina tiveram menor porcentagem de gordura abdominal. Concluiu-se que a substituição da DLmetionina pela MHA não afetou o desempenho, que o plano nutricional normal melhorou a conversão alimentar dos frangos de corte e que as fontes de metionina e os planos nutricionais de AAST não influenciaram o rendimento e a composição da carcaça e vísceras de frangos de corte.
\end{abstract}

Palavras-chave: frango de corte, metionina, metionina hidroxianáloga, exigências nutricionais, carcaça

\begin{abstract}
To evaluate the effects of two different nutritional plans for total sulfur amino acids (TSAA), and two methionine sources in the diets of broiler chickens, an experiment was conducted with 840 male broiler line agRoss 308. The treatments were defined by nutritional plans of TSAA (normal and low) and the methionine sources (DL-methionine and methionine hydroxy-analogue - MHA) used in the diets. The experimental design was completely randomized in a factorial scheme $2 X 2$, (two methionine sources $x$ two levels of nutrition plans TSAA). No differences were found between treatments for ration consumption, weight gain, viability, carcass yield, and carcass composition. The broilers that received the diets with the TSAA normal level had better feed conversion, and those that received diets containing DL-methionine had lower abdominal fat percentages. It was concluded that the substitution of DLmethionine by MHA did not affect the performance of broilers, whereas the normal nutrition plan improved feed conversion of broiler chickens as well as methionine sources and TSAA nutritional plans did not influence the carcass yield and carcass composition of broiler chickens.
\end{abstract}

Keywords: broiler, methionine, methionine hydroxy-analogue, nutrient requirements, carcass

Recebido em 10 de fevereiro de 2009

Aceito em 15 de outubro de 2009

Autor para correspondência (corresponding author)

E-mail: silvana@vet.ufmg.br

Apoio: FAPEMIG 


\section{INTRODUÇÃO}

Os aminoácidos sulfurados totais, metionina e cistina, são essenciais para o crescimento, reações de metilação e síntese de penas e são também importantes precursores da glutationa, taurina e coenzima A (Lehninger et al., 2002). Devido ao importante papel que desempenham no organismo animal, são utilizados em grande quantidade pelas aves durante o período de crescimento. Deste modo, as rações para frangos devem ser suplementadas com aminoácidos sintéticos, disponíveis no mercado. Comumente, são utilizadas duas fontes de metionina sintética: DL-metionina, disponível na forma de pó (DLmetionina) ou na forma líquida como sal de sódio (DL-metionina-Na) e metionina hidroxianáloga (MHA), que se apresenta na forma de pó, como sal de cálcio (MHA-Ca), ou na forma líquida, como ácido livre (MHA-FA). A DL-metionina é usada como padrão para comparar fontes de metionina. Sua atividade é de $99 \%$, enquanto na forma líquida, a atividade de DL-metionina-Na é de $40 \%$. A atividade da MHA é de $88 \%$ para a MHA-FA e de $84 \%$ para a MHA-Ca em relação à DL-metionina. Todas as fontes são produzidas por síntese química. Para produzir a DL-metionina, a mistura deve conter $50 \%$ na forma de D-metionina e $50 \%$ de Lmetionina. A MHA é tipicamente constituída de $65 \%$ de monômeros, $23 \%$ de polímeros e $12 \%$ de água (Lawson e Ivey, 1986). As aves conseguem utilizar apenas a forma $\mathrm{L}$ dos aminoácidos para a síntese proteica e outros processos fisiológicos. Então, as formas D devem ser convertidas para serem usadas pelo organismo animal.

A adição de metionina sob a forma sintética constitui fator preponderante para redução de custo das rações. Com o aparecimento dos MHA, surgiram controvérsias quanto à equivalência em relação a DL-metionina. De acordo com Daenner e Bessei (2003), Visentini et. al (2005), Amarante Junior et al. (2005), Hoehler et al (2005) e Payne et al (2006), a suplementação de rações para frangos de corte com de MHA-FA $(88 \%)$ ou com DL-metionina, no mesmo nível, não tem efeito sobre o desempenho e rendimento de carcaça. Bunchasak e Keawarun (2006), ao compararem MHA-FA (80\%) com DLmetionina, também não encontraram diferenças entre as fontes quanto ao desempenho e rendimento de carcaça. Contudo, Meirelles et al. (2003) observaram melhor conversão alimentar para aves que receberam DL-metionina ao invés de MHA-FA (88\%).

A exigência de aminoácidos sulfurados totais (AAST) para frangos de corte, de um a 21 dias de idade, foram estimadas em 0,898\% (Oliveira Neto et al., 2000), ou 0,907\% (Atencio et al., 2004) e ou 0,931\% (Rostagno et al., 2005). De acordo com Silva Junior et al. (2005), para frangos de corte machos (Ross) de um a 21 dias de idade, a exigência de AAST é de 0,960\%. Lumpkins et al. (2007), em um estudo com frangos da linhagem Cobb 500 de sete a 19 dias de idade, concluíram que a exigência de AAST, independentemente do sexo, foi de $0,892 \%$.

$\mathrm{Na}$ fase de crescimento, a exigência de AAST para frangos de corte é de $0,896 \%$ para machos e de $0,856 \%$ para fêmeas (Rodrigueiro et al., 2000). Segundo Whitaker et al. (2002) e Lumpkins et al. (2007), a exigência de AAST para ambos os sexos é de $0,720 \%$ nessa fase de crescimento e, de acordo com Atencio et al. (2004) é de 0,844\%. Para Quentin et al. (2005) e para Silva Junior et al. (2005), a exigência é de $0,890 \%$.

Com base nesses aspectos, o objetivo deste trabalho foi verificar os efeitos de dois planos nutricionais (normal e reduzido) de AAST e de duas fontes de metionina (DL-metionina e MHA) na ração sobre o desempenho, rendimento e composição de carcaça de frangos de corte de um a 42 dias de idade.

\section{MATERIAL E MÉTODOS}

Foram utilizados 840 pintos de corte, machos, da linhagem agRoss 308, de um dia de idade, alojados em um galpão experimental com 35 aves por boxe (12 aves $\left./ \mathrm{m}^{2}\right)$. O período de criação foi de um a 42 dias de idade, e água e ração foram oferecidas à vontade, durante todo $\mathrm{o}$ período experimental.

O programa de luz utilizado foi: de um a cinco dias de idade, 24 horas de luz por dia, e de seis a 42 dias de idade, somente luz natural conforme recomendado por Moraes et al. (2008).

Foram utilizados três tipos de ração peletizadas, de acordo com a fase de criação, ou seja, inicial de um a 21, crescimento I - de 22 a 37 e crescimento II - de 38 a 42 dias de idade. A composição e os níveis nutricionais da ração de 


\section{Leite et al.}

crescimento II foram os mesmos da ração de crescimento I, porém sem adição de anticoccidianos e promotores de crescimento. Os tratamentos (Tab. 1) foram definidos pelos planos nutricionais, normal e reduzido, de AAST e pelas fontes de metionina utilizadas, DLmetionina (98\% de disponibilidade) e MHA $(82 \%$ da equivalência em relação à DLmetionina). A composição das rações foi isonutritiva, com exceção dos níveis de metionina, e seus respectivos níveis nutricionais, calculados segundo Rostagno et al. (2000), encontram-se nas Tab. 2 e 3, respectivamente.

O desempenho das aves foi avaliado pelo ganho de peso, consumo de ração, conversão alimentar e viabilidade. Para tal, utilizaram-se seis repetições de 35 aves cada por tratamento.

Tabela 1. Descrição dos tratamentos de acordo com os planos, fontes de metionina e fases de criação

\begin{tabular}{ccccccc}
\hline \multirow{2}{*}{ Tratamento } & \multirow{2}{*}{ Plano } & \multicolumn{2}{c}{ Inicial } & \multicolumn{2}{c}{ Crescimento I E II } \\
\cline { 3 - 6 } & & AAST\% Fonte & \multicolumn{2}{c}{ AAST\% Fonte } \\
\hline A & Normal & 0,90 & MHA & 0,88 & MHA \\
B & Normal & 0,90 & DLM & 0,88 & DLM \\
C & Reduzido & 0,85 & DLM & 0,83 & DLM \\
D & Reduzido & 0,85 & MHA & 0,83 & MHA
\end{tabular}

AAST: Aminoácidos sulfurados totais; DLM: DL-metionina; MHA: Metionina hidroxianáloga.

Tabela 2. Composição percentual e níveis nutricionais calculados das rações iniciais

\begin{tabular}{|c|c|c|c|c|}
\hline Ingrediente & Tratamento A & Tratamento B & Tratamento $\mathrm{C}$ & Tratamento D \\
\hline Milho & 60,16 & 60,16 & 60,16 & 60,16 \\
\hline Farelo de soja $46 \%$ & 33,62 & 33,62 & 33,62 & 33,62 \\
\hline Farinha de carne $39 \%$ & 3,47 & 3,47 & 3,47 & 3,47 \\
\hline Gordura de frango & 0,65 & 0,65 & 0,65 & 0,65 \\
\hline MHA $82 \%$ & 0,27 & & & 0,21 \\
\hline DLM (98\%) & 0 & 0,22 & 0,17 & 0 \\
\hline L-lisina & 0,07 & 0,07 & 0,07 & 0,07 \\
\hline L-treonina $98 \%$ & 0,02 & 0,02 & 0,02 & 0,02 \\
\hline Calcário & 0,82 & 0,82 & 0,82 & 0,82 \\
\hline Inerte & & 0,04 & 0,09 & 0,06 \\
\hline Sal comum & 0,40 & 0,40 & 0,40 & 0,40 \\
\hline Supl. vit. mineral $^{1}$ & 0,50 & 0,50 & 0,50 & 0,50 \\
\hline Total $(\%)$ & 100,00 & 100,00 & 100,00 & 100,00 \\
\hline \multicolumn{5}{|l|}{ Nível nutricional } \\
\hline $\mathrm{PB}(\%)$ & 22,06 & 22,06 & 22,06 & 22,06 \\
\hline EM (kcal/kg) & 2950 & 2950 & 2950 & 2950 \\
\hline Cálcio $(\%)$ & 0,92 & 0,92 & 0,92 & 0,92 \\
\hline P disponível (\%) & 0,45 & 0,45 & 0,45 & 0,45 \\
\hline Lisina total $(\%)$ & 1,26 & 1,26 & 1,26 & 1,26 \\
\hline Metionina total $(\%)$ & 0,55 & 0,55 & 0,50 & 0,50 \\
\hline Met + cis total $(\%)$ & 0,90 & 0,90 & 0,85 & 0,85 \\
\hline Sódio $(\%)$ & 0,20 & 0,20 & 0,20 & 0,20 \\
\hline
\end{tabular}

${ }^{C}$ Composição por quilo= vit. A: $13.685 \mathrm{UI}$; vit. D3: $3.157 \mathrm{UI}$; vit. E: $35 \mathrm{mg}$; vit. K3: 4.410mg; vit. B1: $2.415 \mathrm{mg}$; vit. B2 8,6mg; vit. B6: 5.460mg; vit. B12: $21.315 \mathrm{mg}$; biotina: $96.250 \mathrm{mg}$; niacina: $53.900 \mathrm{mg}$; ácido fólico: $1.228,5 \mathrm{mg}$; ácido pantotênico: 13.860mg; colina: 1.760,08mg; selênio: 297,5mg; iodo: $1.000 \mathrm{mg}$; ferro: $30.000 \mathrm{mg}$; cobre: $10.000 \mathrm{mg}$; manganês: 90.000mg; zinco: 80.000mg; BHT: 19.250mg; narasin: 40mg; nicarbazina: 60mg; lincomicina: 13,2mg; ronozyme: $750 \mathrm{FTU}$.

Tratamento $\mathrm{A}=$ plano normal de aminoácidos sulfurados totais (AAST) usando metionina hidroxianáloga (MHA).

Tratamento $\mathrm{B}=$ plano normal AAST usando DL-metionina (DLM).

Tratamento $\mathrm{C}=$ plano reduzido de AAST usando DLM.

Tratamento $\mathrm{D}=$ plano reduzido de AAST usando MHA. 
Efeitos de planos nutricionais...

Tabela 3. Composição percentual e níveis nutricionais calculados das rações de crescimento I e II

\begin{tabular}{lcccc}
\hline Ingrediente & Tratamento A & Tratamento B & Tratamento C & Tratamento D \\
\hline Milho & 53,15 & 53,15 & 53,15 & 53,15 \\
Sorgo & 14,00 & 14,00 & 14,00 & 14,00 \\
Farelo de soja 46\% & 20,80 & 20,80 & 20,80 & 20,80 \\
Farinha de carne 39\% & 1,12 & 1,12 & 1,12 & 1,12 \\
Farinha de vísceras & 2,50 & 2,50 & 2,50 & 2,50 \\
Farinha de penas & 2,00 & 2,00 & 2,00 & 2,00 \\
Farinha de carne suína & 2,00 & 2,00 & 2,00 & 2,00 \\
Gordura de frango & 2,05 & 2,05 & 2,05 & 2,05 \\
MHA 82\% & 0,25 & & & 0,19 \\
DLM (98\%) & & 0,21 & 0,16 & 0,46 \\
Biolys & 0,46 & 0,46 & 0,46 & 0,06 \\
L-treonina 98\% & 0,06 & 0,06 & 0,06 & 0,75 \\
Calcário & 0,75 & 0,75 & 0,75 & 0,09 \\
Inerte & 0,09 & 0,09 & 0,09 & 0,35 \\
Sal comum & 0,35 & 0,35 & 0,35 & 0,50 \\
Supl. vit. min. & 0,50 & 0,50 & 0,50 & 100,00 \\
\hline TOTAL (\%) & 100,00 & 100,00 & 100,00 & 20,35 \\
\hline Nível nutricional & & & & 3150 \\
\hline PB (\%) & 20,35 & 20,35 & 20,35 & 0,90 \\
EM (kcal/kg) & 3150 & 3150 & 3150 & 0,42 \\
Cálcio (\%) & 0,90 & 0,90 & 0,90 & 1,19 \\
P disponível (\%) & 0,42 & 0,42 & 0,42 & 0,44 \\
Lisina total (\%) & 1,19 & 1,19 & 1,19 & 0,83 \\
Metionina total (\%) & 0,49 & 0,49 & 0,44 & 0,20 \\
Met + cis total (\%) & 0,88 & 0,88 & 0,20 & \\
Sódio (\%) & 0,20 & 0,20 & & \\
\hline Composç por quin & & & \\
\hline
\end{tabular}

${ }^{1}$ Composição por quilo= vit. A: 9.775UI; vit. D3: 2.255UI; vit. E: 25mg; vit. K3: 3.150mg; vit. B1: 1.725mg; vit. B2 6,2mg; vit. B6: 3.900mg; vit. B12: $15.225 \mathrm{mg}$; biotina: $68.750 \mathrm{mg}$; niacina: $38.500 \mathrm{mg}$; ácido fólico: $877,5 \mathrm{mg}$; ácido pantotênico: $9.900 \mathrm{mg}$; colina: $1.546,3 \mathrm{mg}$; selênio: $212,5 \mathrm{mg}$; iodo: $1.000 \mathrm{mg}$; ferro: $30.000 \mathrm{mg}$; cobre: $10.000 \mathrm{mg}$; manganês: 90.000mg; zinco: 80.000mg; BHT: 13.750mg; salinomicina: 66mg; lincomicina: 13,2mg; ronozyme: 750FTU.

Tratamento A = plano normal de aminoácidos sulfurados totais (AAST) usando metionina hidroxianáloga (MHA).

Tratamento B = plano normal AAST usando DL-metionina (DLM).

Tratamento $\mathrm{C}=$ plano reduzido de AAST usando DLM.

Tratamento $\mathrm{D}=$ plano reduzido de AAST usando MHA.

Para avaliar o rendimento de carcaça e de cortes, foram abatidos 24 frangos por tratamento e cada ave foi considerada uma repetição, e o abate ocorreu aos 43 dias de idade. Os frangos foram identificados e submetidos ao período ideal de jejum de ração de 10 horas (Schettino et al., 2006), quando foram individualmente pesados na plataforma do abatedouro e abatidos.

Para avaliação do rendimento de carcaça, foi considerado o peso da carcaça eviscerada (com pés, cabeça e pescoço) em relação ao peso vivo em jejum obtido antes do abate. Na avaliação dos demais cortes, coxa e sobrecoxa, peito, dorso, asa, pés/cabeça/pescoço, o rendimento foi considerado em relação ao peso da carcaça eviscerada. A gordura abdominal foi extraída e pesada, e a porcentagem de gordura abdominal foi calculada em relação ao peso da carcaça eviscerada.

Para avaliação da composição da carcaça, foram coletadas seis carcaças com as respectivas vísceras (papo, proventrículo, moela, intestinos delgado e grosso) por tratamento, que foram congeladas $\left(-18^{\circ} \mathrm{C}\right)$ e moídas individualmente. Foram realizadas as análises laboratoriais de matéria seca a $105^{\circ} \mathrm{C}$, proteína bruta, extrato etéreo e matéria mineral (Instrução..., 1999). Os fígados foram colhidos, processados e analisados separadamente. 
O delineamento experimental foi inteiramente ao acaso, em arranjo fatorial $2 \times 2$ (duas fontes de metionina $\mathrm{x}$ dois planos nutricionais de AAST). Para a avaliação do desempenho, foram utilizadas seis repetições de 35 aves por tratamento, para a avaliação do rendimento de carcaça e seus cortes, 24 repetições de uma ave cada, e para as análises de composição das carcaças, vísceras e fígado seis repetições de uma carcaça cada. Para comparação entre médias, usou-se o teste Student Newman Keuls, segundo Sampaio (2002). As análises estatísticas foram realizadas utilizando-se o programa SAS/1996.

\section{RESULTADOS E DISCUSSÃO}

Não houve efeito da interação fontes de metionina versus plano nutricional de AAST, para nenhuma das variáveis avaliadas no desempenho $(\mathrm{P}>0,05)$.

O consumo de ração e o ganho de peso de um a 42 dias de idade das aves não foram influenciados $(\mathrm{P}>0,05)$ pela fonte ou plano nutricional (Tab. 4). Com relação às fontes de metionina, este comportamento está de acordo com o observado por Meirelles et al. (2003) e Visentini et al (2005), que também não verificaram diferenças entre as fontes de metionina. Quanto aos planos nutricionais, os resultados assemelham-se aos de Whitaker et al. (2002) e Amarante Junior et al. (2005). Os trabalhos de Rodrigueiro et al. (2000) e de Silva Junior et al. (2005) revelaram que o aumento do nível de AAST resultou em diminuição do consumo de ração.

Não foi observado efeito da fonte de metionina $(\mathrm{P}>0,05)$ sobre a conversão alimentar em aves de um a 42 dias de idade (Tab. 4). Estes resultados foram semelhantes aos de Visentini et al (2005) e Bunchasak e Keawarun (2006). Porém, Meirelles et al. (2003), ao compararem as duas fontes de metionina (DL-metionina e MHA - 88\%) para frangos de corte, encontraram melhor conversão alimentar para as aves que receberam a DLmetionina. A melhor conversão alimentar $(\mathrm{P} \leq 0,05)$ foi observada nas aves que receberam o plano nutricional normal. Estes resultados estão de acordo com os de Rodrigueiro et al. (2000) que verificaram uma exigência de $0,89 \%$ de AAST para a melhor conversão alimentar. Whitaker et al. (2002) não observaram efeito dos níveis de AAST da dieta sobre a conversão alimentar.

A fonte de metionina e o plano nutricional não tiveram efeito significativo $(\mathrm{P}>0,05)$ sobre a viabilidade das aves (Tab. 4), semelhante ao já observado por Bunchasak e Keawarun (2006).

Tabela 4. Consumo de ração (g), ganho de peso (g), conversão alimentar ( $\mathrm{kg} / \mathrm{kg})$ e viabilidade (\%) de aves de um a 42 dias de idade, em função da fonte e do plano nutricional

\begin{tabular}{lcccc}
\hline Fonte & Consumo de ração & Ganho de peso & Conversão alimentar & Viabilidade \\
\hline DLM & $5393 \mathrm{a}$ & $3285 \mathrm{a}$ & $1,642 \mathrm{a}$ & $95,0 \mathrm{a}$ \\
MHA & $5334 \mathrm{a}$ & $3272 \mathrm{a}$ & $1,630 \mathrm{a}$ & $92,9 \mathrm{a}$ \\
\hline Plano & & & & $93,3 \mathrm{a}$ \\
\hline Normal & $5356 \mathrm{a}$ & $3291 \mathrm{a}$ & $1,627 \mathrm{a}$ & $94,5 \mathrm{a}$ \\
\hline Reduzido & $5371 \mathrm{a}$ & $3266 \mathrm{a}$ & $1,645 \mathrm{~b}$ & 3,55 \\
\hline CV $(\%)$ & 1,92 & 1,64 & 1,07 & \\
\hline
\end{tabular}

Médias seguidas de letras distintas, para fonte e plano, na coluna diferem entre si $(\mathrm{P} \leq 0,05)$.

DLM: DL-metionina (98\%); MHA: metionina hidroxianáloga (82\%).

$\mathrm{CV}=$ coeficiente de variação.

Não foram observados efeitos dos tratamentos $(\mathrm{P}>0,05)$ sobre o rendimento de carcaça inteira, pés, peito, asas e dorso (Tab.5). Com relação às fontes de metionina, os resultados estão de acordo com os de Meirelles et al. (2003), Visentini et al (2005) e Bunchasak e Keawarun (2006), que também não observaram efeito da fonte de metionina sobre o rendimento de carcaças de frangos de corte.
Os frangos que receberam DL-metionina apresentaram menor porcentagem de gordura abdominal $(\mathrm{P} \leq 0,05)$ em relação àqueles que receberam a ração com MHA. Meirelles et al. (2003), Visentini et al (2005) e Bunchasak e Keawarun (2006) não verificaram efeito da fonte de metionina sobre a porcentagem de gordura abdominal. 
Efeitos de planos nutricionais...

Tabela 5. Rendimento de carcaça inteira e de pés, peito, asas, dorso e gordura abdominal (GA) aos 43 dias de idade das aves, em função da fonte e do plano nutricional

\begin{tabular}{lcccccc}
\hline Fonte & Carcaça \% & Pés \% & Peito \% & Asas \% & Dorso \% & GA \% \\
\hline DLM & $81,14 \mathrm{a}$ & $5,14 \mathrm{a}$ & $36,30 \mathrm{a}$ & $8,19 \mathrm{a}$ & $11,39 \mathrm{a}$ & $1,75 \mathrm{a}$ \\
MHA & $80,74 \mathrm{a}$ & $5,19 \mathrm{a}$ & $36,06 \mathrm{a}$ & $8,23 \mathrm{a}$ & $11,08 \mathrm{a}$ & $1,95 \mathrm{~b}$ \\
\hline Planos & & & & & & \\
\hline Normal & $80,81 \mathrm{a}$ & $5,21 \mathrm{a}$ & $36,30 \mathrm{a}$ & $8,19 \mathrm{a}$ & $11,28 \mathrm{a}$ & $1,83 \mathrm{a}$ \\
Reduzido & $81,08 \mathrm{a}$ & $5,12 \mathrm{a}$ & $36,06 \mathrm{a}$ & $8,23 \mathrm{a}$ & $11,18 \mathrm{a}$ & $1,87 \mathrm{a}$ \\
CV $(\%)$ & 1,28 & 7,95 & 4,54 & 6,87 & 9,83 & 21,84 \\
\hline
\end{tabular}

Médias seguidas de letras distintas, para fonte e plano, na coluna diferem entre si $(\mathrm{P} \leq 0,05)$.

DLM: DL-metionina (98\%); MHA: metionina hidroxianáloga (82\%).

$\mathrm{CV}=$ coeficiente de variação.

Para rendimento de coxa e sobrecoxa, foi observado efeito da interação $(\mathrm{P} \leq 0,05)$ fonte versus plano nutricional (Tab. 6). Quando foi utilizado o plano normal não houve diferenças entre as fontes de metionina utilizadas, porém com o plano reduzido de AAST, os frangos que receberam MHA tiveram maior porcentagem de coxa e sobre-coxa do que aqueles que receberam DL-metionina. Estes resultados diferem dos apresentados por Meirelles et al. (2003) e Bunchasak e Keawarun (2006), os quais não encontraram diferenças na porcentagem de coxa e sobrecoxa de frangos alimentados com as diferentes fontes. Em relação ao plano nutricional, os resultados foram semelhantes aos encontrados por Rodrigueiro et al. (2000), Whitaker et al. (2002), Atencio et al. (2004) e Amarante Junior (2005).

Tabela 6. Porcentagem de coxa e sobrecoxa aos 43 dias de idade das aves, em função do plano nutricional e da fonte de metionina

\begin{tabular}{cccc}
\hline \multirow{2}{*}{ Fonte } & \multicolumn{2}{c}{ Planos } & \multirow{2}{*}{ Média } \\
\cline { 2 - 3 } & Normal & Reduzido & 29,07 \\
DLM & $29,45 \mathrm{Aa}$ & $28,69 \mathrm{Ba}$ & 29,37 \\
\hline MHA & $29,21 \mathrm{Aa}$ & $29,54 \mathrm{Aa}$ & \\
\hline Média & 29,34 & 29,12 & \\
\hline
\end{tabular}

Médias seguidas de letras distintas, maiúsculas na coluna e minúsculas na linha diferem entre si $(\mathrm{P} \leq 0,05)$.

DLM - DL-metionina (98\%); MHA - metionina hidroxianáloga (82\%).

$\mathrm{CV}=4,41 \%$.

Não foram observados efeitos da fonte de metionina e nem da porcentagem de AAST sobre a composição de proteína bruta (PB), extrato etéreo (EE) e matéria seca (MS) da carcaça, vísceras e fígado $(\mathrm{P}>0,05)$. Estes resultados estão de acordo com os encontrados por Whitaker et al. (2002). Segundo Rodrigueiro et al. (2000), quando se aumenta a porcentagem de AAST na ração para frangos de corte, ocorre redução da gordura na carcaça. As aves que receberam o plano reduzido de AAST apresentaram maior porcentagem de matéria mineral na carcaça $(\mathrm{P} \leq 0,01)$ em relação àquelas que receberam $\mathrm{o}$ plano normal. $\mathrm{Na}$ literatura, não foram encontradas informações sobre efeito da porcentagem de AAST sobre matéria mineral na carcaça.

Tabela 7. Composição de proteína bruta (PB), extrato etéreo (EE) e matéria mineral (MM) das carcaças, e extrato etéreo (EE) das vísceras e fígados, na matéria seca (MS) aos 43 dias de idade das aves, em função da fonte e do plano nutricional

\begin{tabular}{l|cccccccc}
\hline \multirow{2}{*}{ Fonte } & \multicolumn{9}{c}{ Carcaça } & \multicolumn{2}{c}{ Vísceras } & \multicolumn{2}{c}{ Fígado } \\
\cline { 2 - 9 } & PB & MM & EE & MS & EE & MS & EE & MS \\
\hline DLM & $50,4 \mathrm{a}$ & $6,5 \mathrm{a}$ & $44,4 \mathrm{a}$ & $36,2 \mathrm{a}$ & $56,6 \mathrm{a}$ & $36,2 \mathrm{a}$ & $24,9 \mathrm{a}$ & $28,6 \mathrm{a}$ \\
MHA & $50,7 \mathrm{a}$ & $6,5 \mathrm{a}$ & $44,9 \mathrm{a}$ & $36,1 \mathrm{a}$ & $58,0 \mathrm{a}$ & $36,9 \mathrm{a}$ & $19,8 \mathrm{a}$ & $27,6 \mathrm{a}$ \\
\hline Plano & & & & & & & & $28,3 \mathrm{a}$ \\
\hline Normal & $50,1 \mathrm{a}$ & $5,9 \mathrm{~b}$ & $45,3 \mathrm{a}$ & $36,4 \mathrm{a}$ & $56,8 \mathrm{a}$ & $36,8 \mathrm{a}$ & $23,4 \mathrm{a}$ & $27,8 \mathrm{a}$ \\
Reduzido & $50,9 \mathrm{a}$ & $7,1 \mathrm{a}$ & $44,0 \mathrm{a}$ & $35,9 \mathrm{a}$ & $57,8 \mathrm{a}$ & $36,3 \mathrm{a}$ & $21,3 \mathrm{a}$ & 27,86 \\
\hline CV (\%) & 4,77 & 12,36 & 5,22 & 1,88 & 10,35 & 5,04 & 30,31 & 2,86 \\
\hline
\end{tabular}

Médias seguidas de letras distintas, para fonte e plano, na coluna diferem entre si $(\mathrm{P} \leq 0,05)$.

DLM: DL-metionina (98\%); MHA: metionina hidróxi-análoga (82\%). CV = Coeficiente de variação. 


\section{Leite et al.}

\section{CONCLUSÕES}

A substituição da DL-metionina pela MHA, considerando bioequivalência da MHA em relação à DL-metionina de $82 \%$, não afeta o desempenho de frangos de corte, e o plano nutricional normal de AAST melhora a conversão alimentar dos frangos de corte. As fontes de metionina utilizadas e os planos nutricionais, normal e reduzido, de AAST não influenciam o rendimento e a composição da carcaça e das vísceras de frangos de corte.

\section{AGRADECIMENTOS}

Os autores agradecem o apoio da Fundação de Amparo a Pesquisa do Estado de Minas Gerais FAPEMIG (Belo Horizonte, MG, Brasil) por fornecer fundos para a publicação da pesquisa.

\section{REFERÊNCIAS BIBLIOGRÁFICAS}

AMARANTE JUNIOR, V.S.; COSTA, F.G.P.; BARROS, L.R. et al. Níveis de metionina + cistina para frangos de corte nos períodos de 22 a 42 e de 43 a 49 dias de idade. Rev. Bras. Zootec., v.34, p.1195-1201, 2005.

ATENCIO, A.; ALBINO, L.F.T.; ROSTAGNO, H.S. et al. Exigências de metionina + cistina para frangos de corte machos em diferentes fases de criação. Rev. Bras. Zootec., v.33, p.1152-1166, 2004

BUNCHASAK, C.; KEAWARUN, N. Effect of methionine hydroxy analog-free acid on growth performance and chemical composition of liver of broiler chicks fed a corn-soybean based diet from 0 to 6 weeks of age. Anim. Sci. J., v.77, p.95-102, 2006.

DAENNER, E.; BESSEI, W. Influence of supplementation with liquid DL- methionine hydroxy analogue-free acid (Alimet) or DLmethionine on performance of broilers. J. Appl. Poult. Res., v.12, p.101-105, 2003.

HOEHLER, D.; LEMME, A.; JENSEN, S.K. et al. Relative effectiveness of methionine sources in diets for broiler chickens. J. Appl. Poult. Res., v.14, p.679-693, 2005.

INSTRUÇÃO Normativa ${ }^{\circ} 20$ de 21 de julho de 1999, Métodos analíticos físico-químicos para controle de produtos cárneos e seus ingredientes, sal e salmoura [Ministério da Agricultura e do Abastecimento]. Diário Oficial da União: Brasília em 27 de julho de 1999. Seção 1.
LAWSON, C.Q.; IVEY, F.J. Hydrolylis of 2hydroxy-4 (methylthio) butanoic acid dimer in two model systems. Poult. Sci., v.65, p.1749-1752, 1986.

LEHNINGER, A.L.; NELSON, D.L.; COX, M.M. Princípios da bioquímica. 3.ed. São Paulo: Sarvier, 2002, 975p.

LUMPKINS, B.S.; BATAL, B.S.; BAKER, D.H. Variations in the digestible sulfur amino acid requirement of broiler chickens due to sex, growth criteria, rearing environment, and processing yield characteristics. Poult. Sci., v.86, p.325-330, 2007.

MEIRELLES, H.T.; ALBUQUERQUE, R.; BORGATTI, L.M.O et al. Performance of broilers fed with different levels of methionine hydroxy analogue and DL-methionine. Rev. Bras. Cien. Avic., v.5, p.69-74, 2003.

MORAES, D.T.; LARA, L.J.C.; BAIÃO, N.C. et al. Efeitos dos programas de luz sobre desempenho, rendimento de carcaça e resposta imunológica em frangos de corte. Arq. Bras. Med. Vet. Zootec., v.60, p.201-208, 2008.

OLIVEIRA NETO, A.R.; OLIVEIRA, R.F.M.; DONZELE, J.L.et al. Níveis de metionina + cistina para pintos de corte mantidos em ambiente termoneutro. In: REUNIÃO ANUAL DA SOCIEDADE BRASILEIRA DE ZOOTECNIA, 37., 2000, Viçosa. Anais... Viçosa, 2000. p.310.

PAYNE, R.L.; LEMME, A.; SEKO, H. et al. Bioavailability of methionine hydroxy analog-free acid relative to DL-methionine in broilers. $\mathrm{An}$. Sci. J., v.77, p.427-439, 2006.

QUENTIN, M.; BOUVAREL, I.; PICARD, M. Effects of the starter diet, light intensity, and essential amino acids level on growth and carcass composition of broilers. J. Appl. Poult. Res., v.14, p.69-76, 2005.

RODRIGUEIRO, R.J.B.; ALBINO, L.F.T.; ROSTAGNO, H.S. et al. Exigência de metionina + cistina para frangos de corte na fase de crescimento e acabamento. Rev. Bras. Zootec., v.29, p.507-517, 2000 .

ROSTAGNO, H.S.; ALBINO, L.F.T.; DONZELE, J.L. et al. Tabelas brasileiras para aves e suinos. Composição de alimentos e exigências nutricionais. Viçosa: UFV, 2000, 141p.

ROSTAGNO, H.S.; ALBINO, L.F.T.; DONZELE, J.L. et al. Composição de alimentos e exigências nutricionais de aves e suinos. Tabelas Brasileiras. 2.ed. Viçosa: UFV, 2005. 186p. 
SAMPAIO, I.B.M. Estatística aplicada à experimentação animal. 2.ed. Belo Horizonte: FEPMVZ, 2002. 244p.

SCHETTINO, D.N., CANCADO, S.V., BAIAO, N.C. et al. Efeito do período de jejum pré-abate sobre o rendimento de carcaça de frango de corte. Arq. Bras. Med. Vet. Zootec., v.58, p.918-924, 2006.

SILVA JUNIOR, R.G.C.; LANA, G.R.Q; RABELLO, C.B.V. et al. Exigências de metionina + cistina para frangos de corte machos de 1 a 21 e de 22 a 42 dias de idade, em clima tropical. Rev. Bras. Zootec., v.34, supl.4, p.2399-2407, 2005.
VISENTINI, P.; LOPES, J.; TOLEDO, G.S. et al. Níveis de substituição da DL-metionina pela metionina hidroxianáloga em base equimolar, em dietas para frangos de corte. Cien. Rural, v.35, p.1400-1405, 2005.

WHITAKER, H.M.A; MENDES, A.A.; GARCIA, E.A. et al. Efeito da suplementação de metionina sobre o desempenho e a avaliação de carcaças de frangos de corte. Rev. Bras. Cien. Avic., v.4, p.1-9, 2002. 